\title{
Ultraquantum turbulence in a quenched homogeneous Bose gas
}

\author{
G. W. Stagg, ${ }^{*}$ N. G. Parker, and C. F. Barenghi \\ Joint Quantum Centre (JQC) Durham-Newcastle, School of Mathematics and Statistics, \\ Newcastle University, Newcastle upon Tyne, NE1 7RU, United Kingdom
}

(Received 13 July 2016; published 28 November 2016)

\begin{abstract}
Using the classical field method, we study numerically the characteristics and decay of the turbulent tangle of superfluid vortices which is created in the evolution of a Bose gas from highly nonequilibrium initial conditions. By analyzing the vortex line density, the energy spectrum, and the velocity correlation function, we determine that the turbulence resulting from this effective thermal quench lacks the coherent structures and the Kolmogorov scaling; these properties are typical of both ordinary classical fluids and of superfluid helium when driven by grids or propellers. Instead, thermal quench turbulence has properties akin to a random flow, more similar to another turbulent regime called ultraquantum turbulence, which has been observed in superfluid helium.
\end{abstract}

DOI: 10.1103/PhysRevA.94.053632

\section{INTRODUCTION}

The formation of a coherent Bose-Einstein condensate from a thermal Bose gas is a rich topic of ongoing research [1]. Recent experiments on thermally quenched Bose gases have observed the spontaneous formation of defects in the guise of vortices [2,3] and solitonic vortices [4], confirming the occurrence of the Kibble-Zurek mechanism [5,6] in these gases. A paradigm for this nonequilibrium phase transition is the formation of a homogeneous weakly interacting Bose gas starting from highly nonequilibrium initial conditions [7-15]. The gas is modeled as a classical matter field described by the Gross-Pitaevskii equation. This field undergoes a universal self-ordering into a quasicondensate, i.e., a coherent superfluid component, and a noncondensed, thermal component. Phase dislocations that become entrapped within the quasicondensate during this quench give rise to an irregular tangle of quantized vortex lines that permeate the system, as seen in Fig. 1. This corresponds to a state of superfluid turbulence. Over time, this turbulent state relaxes, tending towards the vortex-free, partially condensed equilibrium state of the finite-temperature Bose gas.

The aim of our work is to explore the nature of this state of superfluid turbulence, including the characteristics of its decay. Is it similar to turbulence in ordinary (classical) fluids? The question is natural, because tangles of quantized vortices created in superfluid helium by moving grids or propellers appear to obey classical scaling laws [17-19].

By examining the distribution of kinetic energy over the length scales, the velocity correlation function, and the temporal decay of the vortex line density, we show that the turbulence resulting from the thermal quench of a Bose gas is very different from classical turbulence and any quasiclassical regimes of superfluid turbulence. Instead, it shares key properties with a second state of turbulence [20,21],

\footnotetext{
*george.stagg@ncl.ac.uk

Published by the American Physical Society under the terms of the Creative Commons Attribution 3.0 License. Further distribution of this work must maintain attribution to the author(s) and the published article's title, journal citation, and DOI.
}

which has also been observed in superfluid helium, called ultraquantum turbulence, which has unusual nonclassical properties.

\section{CLASSICAL FIELD METHOD}

We model a weakly interacting, homogeneous Bose gas (including thermal excitations) by means of the classical field method [7-15]. The gas is parametrized by a classical matter field $\psi(\mathbf{r}, t)$, normalized to the total number of particles $N=\int|\psi|^{2} d V$, and whose evolution follows the GrossPitaevskii equation (GPE):

$$
i \hbar \frac{\partial \psi}{\partial t}=-\frac{\hbar^{2}}{2 m} \nabla^{2} \psi+g|\psi|^{2} \psi .
$$

The cubic term accounts for the repulsive interactions between the particles, with $g=4 \pi \hbar^{2} a_{s} / m$, where $m$ is the particle mass and $a_{s}$ is the interparticle $s$-wave scattering length (here $a_{s}>0$ ). The total energy of the gas is

$$
H=\int\left(\frac{\hbar^{2}}{2 m}|\nabla \psi|^{2}+\frac{g}{2}|\psi|^{4}\right) d V .
$$

The GPE is conventionally used to model a zerotemperature condensate, but it is now established that, provided the modes of the gas are highly occupied, the gas evolves as an ensemble of modes, each of which follows (to leading order) the classical trajectory described by the GPE [22-24]. Various phenomena have been studied within this classical field formalism, including equilibration dynamics [12,13,15,25], critical temperatures [26], correlation functions [27], vortex nucleation [28-31] and decay of vortex rings [32], nonthermal fixed points [33], as well as extensions to binary condensates [34-36].

A classical field $\psi(\mathbf{r}, t)$ representing $N$ particles is simulated in a cubic periodic box of volume $D^{3}$. We make the GPE dimensionless using the natural units of the homogeneous system: Particle number density is expressed in terms of the average value $\rho=\left\langle|\psi|^{2}\right\rangle=N / D^{3}$, length in terms of the healing length $\xi=\hbar / \sqrt{m g \rho}$, speed in terms of the speed of sound $c=\sqrt{\rho g / m}$, energy in terms of the chemical potential $\mu=\rho g$, and time in terms of $\tau=\hbar / g \rho$. The time evolution is computed using a fourth-order Runge-Kutta scheme with time 
(a)

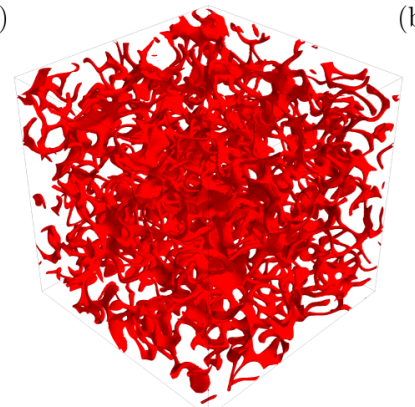

(b)

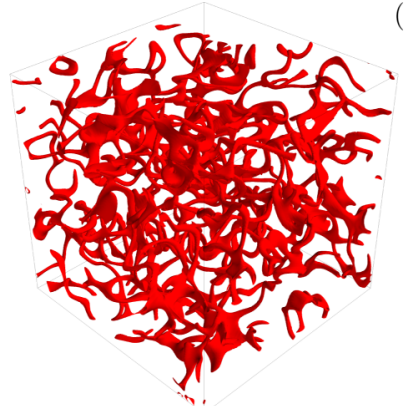

(c)

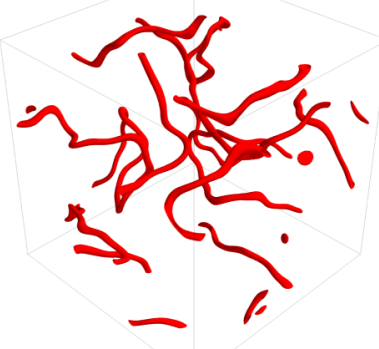

(d)

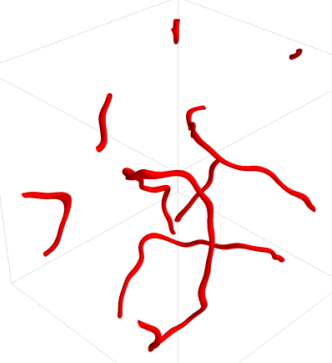

FIG. 1. Sample evolution of the turbulent vortex tangle (present in the condensed part of the Bose gas) as it decays to equilibrium. Here the condensate fraction of the gas is $\rho_{0} / \rho=0.22$. Shown are isosurfaces of the quasicondensate density at isosurface level $0.05\left\langle|\hat{\psi}|^{2}\right\rangle$ at times (a) $t / \tau=0$, (b) 250, (c) 1250, and (d) 2500. At later times (not shown) all vortex lines disappear from the system. Here the quasicondensate is visualized using a cutoff of $k_{c}=10(2 \pi / D)$. In the Supplemental Material [16], we provide a movie of this process. A similar figure and further description of the quasicondensate filtering are shown in Ref. [13].

step $\Delta t=0.01 \tau$ on a $192^{3}$ grid with isotropic grid spacing $d=0.75 \xi$.

To ensure that all numerically accessible modes are highly occupied, the initial condition is the highly nonequilibrium state,

$$
\psi(\mathbf{r}, 0)=\sum_{\mathbf{k}} a_{\mathbf{k}} \exp (i \mathbf{k} \cdot \mathbf{r})
$$

where the $192^{3}$ modes of the system are labeled by the wave vector $\mathbf{k}$, the coefficients $a_{\mathbf{k}}$ are uniform, and the phases are distributed randomly [13]. The occupation of mode $\mathbf{k}$ is $n_{\mathbf{k}}=$ $\left|a_{\mathbf{k}}\right|^{2}$. The spatial grid discretization implies that high momenta are not described; in effect an ultraviolet cutoff is introduced, $n_{\mathbf{k}}(t)=0$ for $k>k_{\max }$, where $k=|\mathbf{k}|$ and the maximum described wave vector amplitude is $k_{\max }=\sqrt{3} \pi / d$ [37]. While our simulations are essentially identical in nature to those conducted by Berloff and Svistunov [13], where the formation of a vortex tangle and its decay over time was noted but not studied in detail, it is the quantitative analysis of this tangle which is the focus of our work. There are also some small technical differences in the implementation of our simulations and analysis, which are stated throughout the discussion below.

The condensate fraction $\rho_{0} / \rho$ of the equilibrium state (where $\rho_{0}$ is the density of the quasicondensate, to be defined in Sec. III A) is uniquely determined by the number density, $N / D^{3}$, and the energy density, $\langle H\rangle / D^{3}$. In practice these values are controlled by a rescaling of the initial condition $\psi(\mathbf{r}, 0)$ so as to modify the uniform value of $n_{\mathbf{k}}$ in the initial condition, with a selection of high momenta coefficients $a_{\mathbf{k}}$ set to zero in order to retain the same number density after rescaling. The process can be seen for the initial condition in Fig. 2.

Our results focus on the three cases: low condensate fraction $\left(\rho_{0} / \rho=0.22\right)$, moderate condensate fraction $\left(\rho_{0} / \rho=0.48\right)$, and high condensate fraction $\left(\rho_{0} / \rho=0.77\right)$. The parameters used to generate these cases are listed in Table I, along with the corresponding temperature of each state, $T / T_{c}$, where $T_{c}$ is the critical temperature for the condensation, estimated using the empirical formula provided in Ref. [32]. The specification of the temperature of the equilibrium states was not performed in the corresponding work of Ref. [13].

\section{FORMATION OF THE TURBULENT VORTEX TANGLE}

\section{A. The quasicondensate}

The evolution of the system in momentum space $[13,36]$ is shown in Fig. 2 (upper). At $t=0$ (red circles), the mode occupation numbers $n_{k}$ are distributed uniformly with $k$, with values of the order of unity, up to the cutoff, as per the imposed initial condition. Over time, self-ordering of the classical field leads to a smoothing of the mode occupation distribution towards a characteristic bimodal form. At low $k$ the modes have macroscopic occupation $\left(n_{k} \gg 1\right)$, characteristic of Bose-Einstein condensation and of the presence of the quasicondensate. At high $k$ the modes have low occupation and are the thermal excitations. Their distribution approximately follows the Rayleigh-Jeans equilibrium distribution for noninteracting particles, $n_{k} \sim k^{-2}$ [38]. The transition in $k$ space between these two components is most evident when viewing the integral distribution of the particles over the wave numbers, $F_{k}=\sum_{k^{\prime}<k} n_{\mathbf{k}^{\prime}}$ (inset of Fig. 2); here a prominent shoulder in the curve marks the transition from the quasicondensate to the thermal modes.

To allow us to analyze the coherent superfluid structures which form, we must identify the quasicondensate component of the gas. This is most readily distinguished from the thermal component in $k$ space, and we denote the boundary between these two components as the cutoff wave number, $k_{c}$. The shoulder which forms in the integral distribution $F_{k}$ provides a natural definition for this boundary. The position of the shoulder shifts with time, rapidly at first and then slowing towards an equilibrium value $k \xi \approx 0.2$. One can take this shift into account, as per Ref. [13], by empirically modeling the shift of the cutoff $k_{c}$ in time. However, the vortex tangle in which we are interested takes a short time (approximately 200 $\tau$ ) to emerge from the noise and by the time this has happened the shoulder has almost reached its equilibrium position. As such, we define the cutoff to be fixed in time, and focus on the value $k_{c 1}=10(2 \pi / D) \approx 0.43 \xi^{-1}$. This value is the approximate position of the shoulder by the time the tangle has formed $(\approx 200 \tau)$. At later time, when the shoulder converges to 

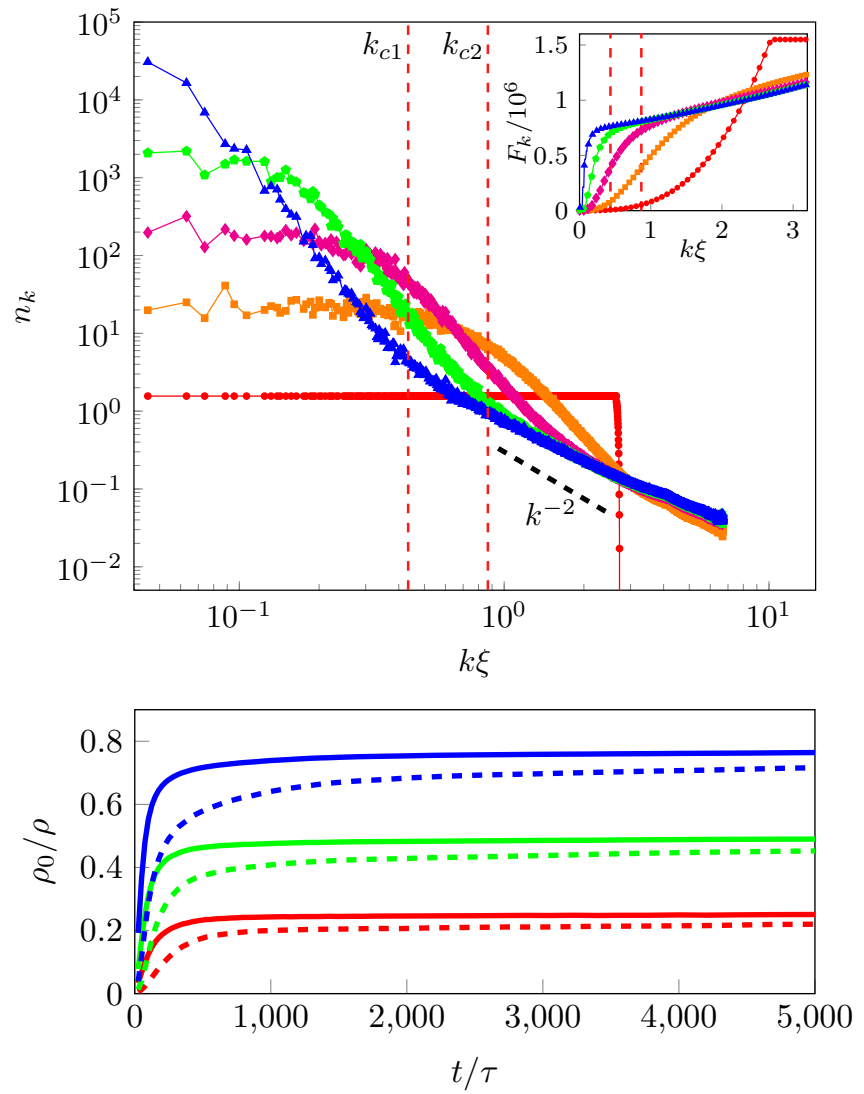

FIG. 2. Upper: Occupation numbers $n_{k}$ as a function of wave number $k \xi$ at different times $t / \tau$ as the Bose gas decays to equilibrium: $t / \tau=0$ (red circles), $t / \tau=25$ (orange squares), $t / \tau=75$ (magenta diamonds), $t / \tau=200$ (green pentagons), and $t / \tau=1000$ (blue triangles). Here the final condensate fraction at equilibrium is $\rho_{0} / \rho=0.48\left(\langle H\rangle / D^{3}=1.33\right)$. A line proportional to the RayleighJeans distribution, $n_{k} \propto k^{-2}$, is shown by a black dashed line as a guide to the eye. Inset: Integral distribution of the particles, $F_{k}=\sum_{k^{\prime}<k} n_{\mathbf{k}^{\prime}}$ vs $k \xi$ at the same times as in the main figure. In both the main figure and the inset $k_{c 1}=10(2 \pi / D)$ and $k_{c 2}=20(2 \pi / D)$ are labeled by red dashed lines. Lower: Condensate fraction over time for the quasicondensate filtered with a cutoff of $k_{c}=10(2 \pi / D)$ (dashed lines) and $k_{c}=20(2 \pi / D)$ (solid lines), for energy densities $\langle H\rangle / D^{3}=2.13$ (lower red/intermediate gray lines), $\langle H\rangle / D^{3}=1.33$ (middle green/light gray lines), and $\langle H\rangle / D^{3}=0.53$ (upper blue/dark gray lines). We find our choices of $k_{c}$ lead to only minimal variation of $\rho_{0} / \rho$ at equilibrium.

slightly lower wave number, this fixed cutoff will encapsulate a small, but effectively negligible, fraction of the thermal component. We also consider a second cutoff, $k_{c 2}=20(2 \pi / D)$, and find that our results are insensitive to the value chosen, thereby validating this definition of quasicondensate.

Due to the randomized phases in the initial condition, the system is full of phase defects, and a dense vortex tangle forms in the quasicondensate, as seen in Fig. 1(a). The raw field $\psi$ is too noisy to directly visualize the superfluid vortex tangle. Following Ref. [13], this problem is overcome by defining a quasicondensate field $\hat{\psi}$ via $\hat{a}_{\mathbf{k}}=a_{\mathbf{k}} \times \max \left\{1-k^{2} / k_{c}^{2}, 0\right\}$. This procedure, which filters high-frequency modes from $\psi$, is analogous to spatial course-grained averaging, so that $\hat{\psi}$
TABLE I. The initial condition parameters and the resulting condensate fraction $\rho_{0} / \rho$ and mean occupation number of the lowest momentum mode $n_{k=0}$ (averaged over 100 time steps) of the equilibrium state of the Bose gas. The quoted condensate fractions are calculated based on the choice of cutoff wave number $k_{c}=k_{c 1}$. Also shown is the temperature of the gas, $T / T_{c}$, estimated using the empirical formula in Ref. [32].

\begin{tabular}{lccc}
\hline \hline \multicolumn{4}{c}{ Initial conditions } \\
\hline$N / D^{3}\left(\xi^{-3}\right)$ & 0.50 & 0.50 & 0.50 \\
$\langle H\rangle / D^{3}\left(\mu \xi^{-3}\right)$ & 2.13 & 1.33 & 0.53 \\
\multicolumn{5}{c}{$\rho_{0} / \rho$} & Equilibrium state & & \\
$\left\langle n_{k=0} / N\right\rangle$ & 0.22 & 0.48 & 0.77 \\
$T / T_{c}$ & 0.21 & 0.46 & 0.72 \\
\hline \hline
\end{tabular}

contains only the long-wavelength component of the classical field $\psi$. The quasicondensate density is then $|\hat{\psi}|^{2}$ and its value is $\rho_{0}=\left\langle|\hat{\psi}|^{2}\right\rangle$; the condensate fraction follows as $\rho_{0} / \rho$, i.e., the ratio of the quasicondensate density to the total density. Whereas in Ref. [13] the cutoff $k_{c}$ was chosen to vary in time, here we find it sufficient to use a fixed value (and we confirm that our results are insensitive to the precise value chosen).

The evolution of the condensate fraction is shown in Fig. 2 (lower). The condensate fraction $\rho_{0} / \rho$ is approximately zero at $t=0$. It immediately undergoes a rapid growth as the quasicondensate forms. The growth then slows and asymptotes towards its equilibrium value. For our primary choice of cutoff, $k_{c}=k_{c 1}$, the condensate fraction reaches $95 \%$ of its asymptotic value within a time $t=600 \tau$; in comparison, the decay of the vortex tangle towards the vortex-free state typically occurs on a timescale of $t=7000 \tau$. For the second choice of cutoff, $k_{c}=k_{c 2}$, the growth of the condensate fraction is slightly faster and the final condensate fraction is slightly larger, but the qualitative behavior is unchanged. We have verified that while our simulations are sensitive to the initial condition parameters shown in Table I, the behaviors demonstrated in Fig. 2 are consistent over different randomized initial conditions.

The vortices are visualized, such as in Fig. 1, through isosurface plots of the quasicondensate density. The isosurface density level varies with time according to $|\hat{\psi}|^{2}=0.05\left\langle|\hat{\psi}|^{2}\right\rangle$. This level is chosen so that the resulting vortex structures are depicted with approximately constant cross-sectional radius throughout the decay, and this is similar to the radius of a vortex core at zero temperature.

\section{B. Energy spectrum}

One method to characterize the turbulence of the vortex tangle formed in the quasicondensate is by studying how the kinetic energy of the vortices is distributed over wave number. There are three wave-number scales of particular interest in this problem: $k_{D}=2 \pi / D$ (associated with the length scale of the computational box), $k_{\xi}=2 \pi / \xi$ (associated with the superfluid healing length), and $k_{\ell}$ (associated with the typical intervortex spacing, $\ell$ ). The typical intervortex spacing can be estimated as $\ell \approx 1 / \sqrt{L}$, where $L$ is the vortex line density 


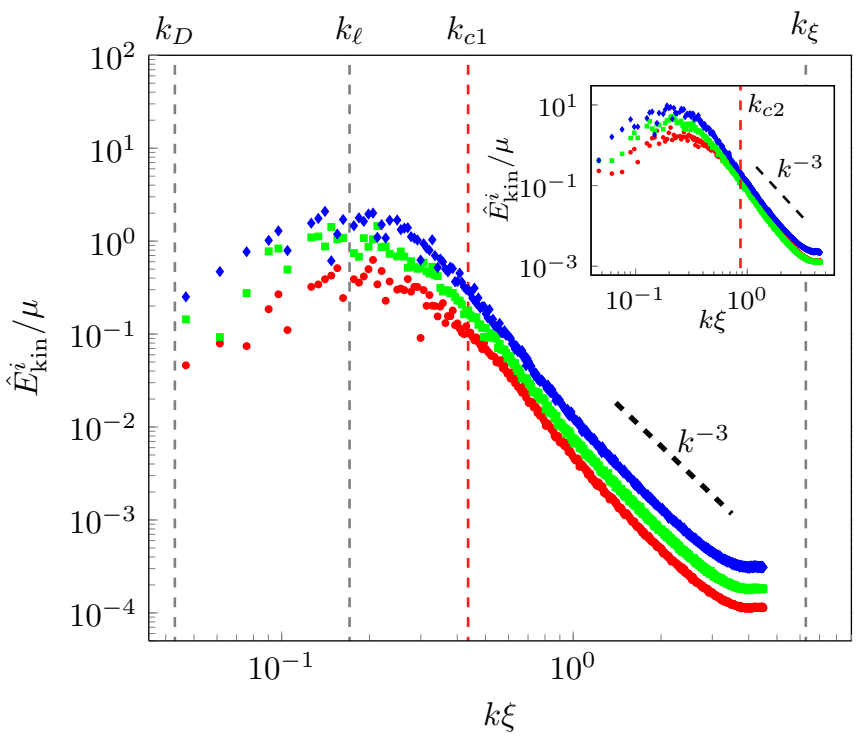

FIG. 3. Incompressible kinetic energy spectrum of the quasicondensate for different temperatures, corresponding to condensate fraction $\rho_{0} / \rho=0.22$ (red/intermediate gray circles), $\rho_{0} / \rho=0.48$ (green/light gray squares), and $\rho_{0} / \rho=0.77$ (blue/dark gray diamonds) at time $t / \tau=300$, and with a cutoff of $k_{c}=10(2 \pi / D)$ (marked by a dashed line labeled $k_{c 1}$ ). Dashed lines also mark wave numbers $k_{D}, k_{\ell}$, and $k_{\xi}$ corresponding to the length scale of the box, the typical inter-vortex spacing, and the healing length, respectively. A black dashed line with $k^{-3}$ dependence is plotted as a guide to the eye. Inset: The same simulations, but with a quasicondensate cutoff of $k_{c}=20(2 \pi / D)$ (marked by a dashed line labeled $\left.k_{c 2}\right)$.

(length of vortex line per unit volume). These scales provide the perspective required to interpret the distribution of energy.

The kinetic energy density of the quasicondensate is defined as $E_{\text {kin }}=\frac{\hbar^{2}}{2 m}|\nabla \hat{\psi}|^{2}$. Using Parseval's theorem, the corresponding kinetic energy spectrum, $\hat{E}_{\text {kin }}(k)$, can be written in terms of the angle average of $\left|\mathcal{F}\left(\sqrt{E_{\text {kin }}}\right)\right|^{2}$ [39], where $\mathcal{F}$ denotes the Fourier transform, so that

$$
\int E_{\mathrm{kin}}(\mathbf{r}) d V=\int \hat{E}_{\mathrm{kin}}(k) d k
$$

The kinetic energy can be further decomposed into compressible and incompressible parts, $E_{\mathrm{kin}}=E_{\mathrm{kin}}^{i}+E_{\mathrm{kin}}^{c}$, where the compressible part is associated with sound waves and the incompressible part with vortices. This decomposition can be defined through the hydrodynamic interpretation of the superfluid as $\sqrt{n} \mathbf{v}=(\sqrt{n} \mathbf{v})^{c}+(\sqrt{n} \mathbf{v})^{i}$ with $\nabla \cdot(\sqrt{n} \mathbf{v})^{i}=0$ and $\nabla \times(\sqrt{n} \mathbf{v})^{c}=0$, where $n=\left|\psi^{2}\right|$ is the particle density and $\mathbf{v}$ is the superfluid velocity. The incompressible kinetic energy spectrum is then denoted $\hat{E}_{\text {kin }}^{i}(k)$, defined in a way similar to $\hat{E}_{\text {kin }}(k)$ in Eq. (4).

Figure 3 shows the incompressible energy spectrum of the vortex tangle at time $t / \tau=300$ (after the quasicondensate and tangle have formed but before the turbulence has undergone any significant decay). This spectrum clearly shows that our turbulence is unlike turbulence in ordinary fluids. In classical turbulence, the energy is concentrated at the smallest wave number $k_{D}$, decreasing as $k^{-5 / 3}$ (the celebrated Kolmogorov scaling) in the region $k_{D} \ll k \ll k_{\ell}$. Figure 3 shows no such

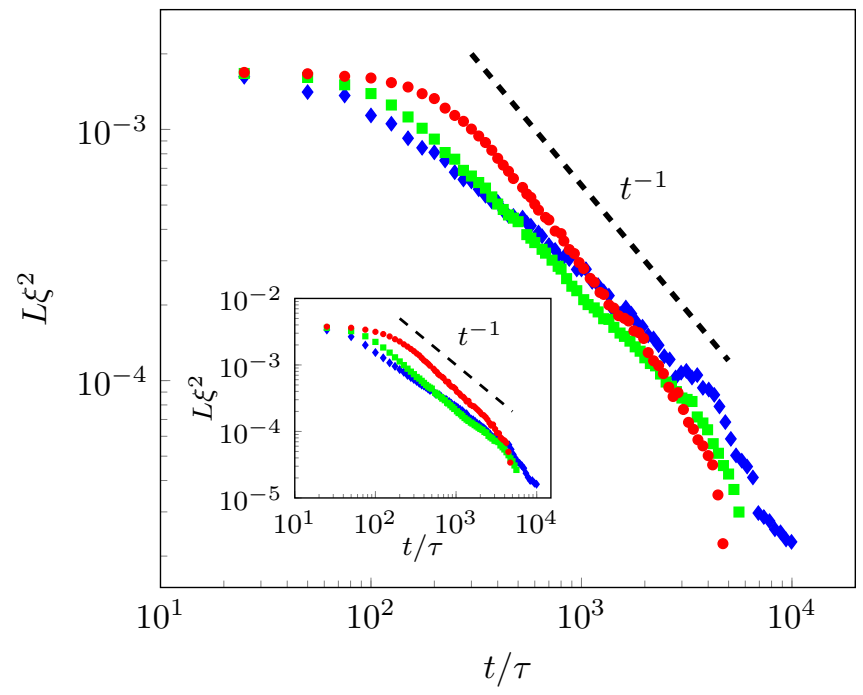

FIG. 4. Vortex line density $L$ over time $t$ for condensate fraction $\rho_{0} / \rho=0.22$ (red/intermediate gray circles), $\rho_{0} / \rho=0.48$ (green/light gray squares), and $\rho_{0} / \rho=0.77$ (blue/dark gray diamonds). Each line is an average of five simulations. The line proportional to $t^{-1}$ (characteristic of the decay of ultraquantum turbulence) is shown (dashed black line) as a guide to the eye. Inset: The same simulations, but with a quasicondensate cutoff of $k_{c}=20(2 \pi / D)$.

pileup of energy near $k_{D}$ and no Kolmogorov scaling; on the contrary, the energy peaks at length scales just above $k_{\ell}$. The visible $k^{-3}$ dependence of the spectra in the region $k_{\ell}<k<k_{\xi}$ arises from the vortex cores [39]. Note that these results are insensitive to the cutoff; we see the same behavior for our second choice of cutoff $k_{c}=k_{c 2}$ (inset of Fig. 3).

\section{RELAXATION OF THE TURBULENT VORTEX TANGLE}

During the decay of the superfluid turbulence, shown in Fig. 1, the vortex tangle remains random and isotropic throughout its decay to one or more vortex lines or rings, which eventually also decay, leading to a vortex-free state. Insight into the nature of the turbulent decay is obtained by monitoring the vortex line density $L$, defined as the vortex length per unit volume; the vortex length is estimated as $V_{\mathrm{t}} / A$ where $V_{\mathrm{t}}$ is the total volume within isosurface vortex tubes, and $A$ is the circular cross-sectional area of a vortex, which is constant for a steady condensate fraction.

Figure 4 shows the decay of the vortex line density over time. At very early times $(t \lesssim 300 \tau)$ the tangle is in the process of forming, while at very late times $(t \gtrsim 3000 \tau)$ only one or two vortices or vortex rings remain. However, in the large intervening range of time a sizable tangle exists. During this range the decay of the vortex line density has a power-law form, $L=\alpha t^{-\beta t}$ with $\alpha$ constant and $\beta \approx 1$ (or slightly less depending on the condensate fraction). For comparison, the decay of classical Kolmogorov turbulence is significantly quicker, $L \sim t^{-3 / 2}$ [40-42], and hence Fig. 4 is consistent with Fig. 3 and the absence of a Kolmogorov spectrum in our system. 
TABLE II. Best-fitting power law decay of the vortex line density over time (Fig. 4), fitted to the equation $L(t)=\alpha t^{-\beta}$. The data are fitted over the region indicated by the length of the $L \sim t^{-1}$ guide line. The $95 \%$ confidence interval for each fitting parameter is also indicated.

\begin{tabular}{cccc}
\hline \hline$\rho_{0} / \rho$ & $\alpha$ & $\beta$ & $k_{c}$ \\
\hline 0.22 & $0.40 \pm 0.07$ & $1.04 \pm 0.03$ & $10(2 \pi / D)$ \\
0.48 & $0.26 \pm 0.03$ & $1.03 \pm 0.02$ & $10(2 \pi / D)$ \\
0.77 & $0.07 \pm 0.02$ & $0.83 \pm 0.05$ & $10(2 \pi / D)$ \\
0.22 & $0.59 \pm 0.13$ & $1.04 \pm 0.04$ & $20(2 \pi / D)$ \\
0.48 & $0.28 \pm 0.02$ & $1.05 \pm 0.02$ & $20(2 \pi / D)$ \\
0.77 & $0.07 \pm 0.02$ & $0.84 \pm 0.03$ & $20(2 \pi / D)$ \\
\hline \hline
\end{tabular}

Instead, the exponent $\beta \approx 1$ which we observe (see Table II) is consistent with the ultraquantum turbulent regime revealed by the experiments of Walmsley and Golov [20], who created the turbulence by injecting vortex rings in a sample of superfluid helium initially at rest. In another set of experiments, Walmsley and Golov found that a longer, more intense injection stage generates quasiclassical turbulence which decays as $L \sim t^{-3 / 2}$. Following Volovik [43], Walmsley and Golov argued that whereas quasiclassical turbulence implies the existence of an energy cascade of eddies or vortex bundles [44] similar to ordinary turbulence, ultraquantum turbulence lacks coherent structures and is more akin to a random flow. This interpretation was confirmed by numerical calculations of Baggaley et al. [45], who simulated the experiments, reproducing both ultraquantum $\left(L \sim t^{-1}\right)$ and quasiclassical $\left(L \sim t^{-3 / 2}\right)$ regimes, and verified the presence of a Kolmogorov energy spectrum $\left[\hat{E}_{\text {kin }}(k) \sim k^{-5 / 3}\right]$ only in the latter.

From the fitting parameter $\alpha$, assuming that the energy dissipation rate per unit mass has the classical form $d E / d t=$ $-v \omega^{2}$, where the energy per unit mass, $E$, is proportional to the length of vortex line and the vorticity is estimated as $\omega \approx \kappa L$, we infer that the turbulent kinematic viscosity $v$ is such that $0.06<v / \kappa<0.3$, in fair agreement with numerical $(\nu / \kappa=$ 0.06 and $0.1[45,46])$ and experimental $(\nu / \kappa=0.1[47])$ values for superfluid helium, although our values do not capture the temperature trend in helium.

To confirm the ultraquantum interpretation of the turbulence created by a thermal quench we compute the velocity correlation function, $f(r)$, defined (at fixed time $t$ ) as

$$
f(r, t)=\frac{\left\langle v_{x}(\mathbf{r}, t) v_{x}\left(\mathbf{r}+r \hat{\mathbf{e}}_{x}, t\right)\right\rangle}{\left\langle v_{x}(\mathbf{r}, t)^{2}\right\rangle}
$$

where $\mathbf{v}$ is the velocity of the quasicondensate and the ensemble average is performed over positions $\mathbf{r}$. Since the flow is isotropic, we only present results for displacements along one direction, here chosen to be the $x$ direction. The function $f(r)$ is dimensionless and normalized to $f(0)=1$. If the vortex lines are essentially randomly oriented then at a distance $r \approx \ell / 2$ the velocity correlation should vanish. Indeed, the inset of Fig. 5 shows that at time $t / \tau=300$ (when we monitor the energy spectrum, and $\ell \approx 30 \xi$ ) the correlation function has become negligible at such distances. In fluid dynamics, a convenient measure of the distance over which velocities are correlated, is the integral length scale [48], defined (at time $t$ )

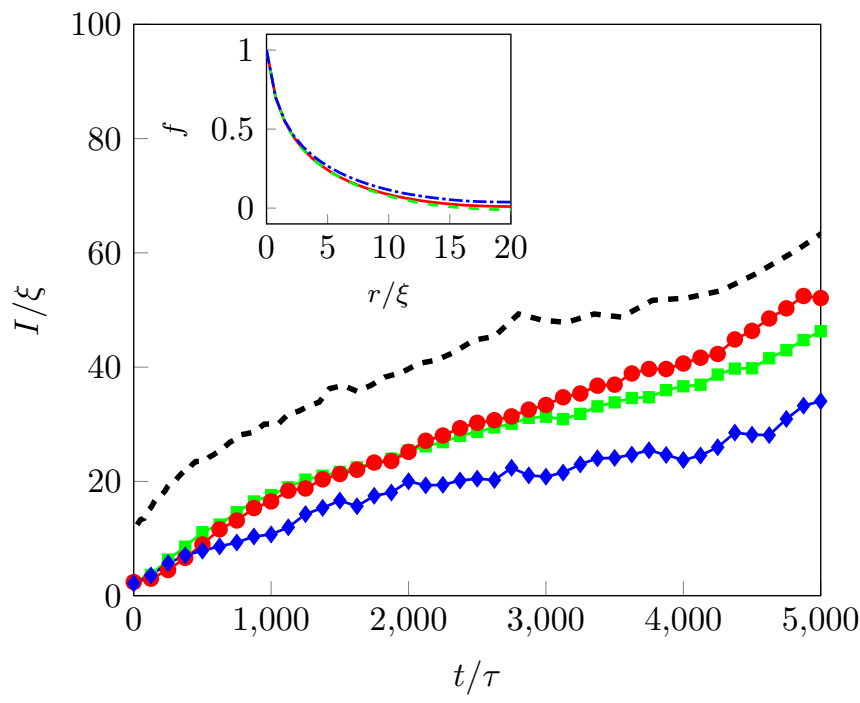

FIG. 5. Integral scale $I$ as a function of time $t$ as the vortex tangle decays, for $\rho / \rho_{0}=0.22$ (red/intermediate gray circles), $\rho / \rho_{0}=0.48$ (green/light gray squares), $\rho / \rho_{0}=0.77$ (blue/dark gray diamonds), and half the typical intervortex spacing $\ell / 2$ (dashed line). Inset: Velocity correlation function $f(r, t)$ at time $t=300 \tau$ for equilibrium states at $\rho / \rho_{0}=0.22$ (red/intermediate gray solid line), $\rho / \rho_{0}=0.48$ (green/light gray dashed line), and $\rho / \rho_{0}=0.77$ (blue/dark gray dot-dashed line).

as

$$
I(t)=\int_{0}^{\infty} f(r, t) d r .
$$

Figure 5 shows $I$ as a function of $t$ during the evolution of the vortex tangle. Clearly $I(0) \approx 0$ at very early times, reflecting the random nature of the initial condition. At later times, $I(t)$ increases for all condensate fractions: As the tangle decays, fewer vortices are left, and the velocity field becomes correlated over larger regions of space. The fact that $I(t)$ remains less than $\ell$ at all confirms the disorganized nature of our turbulence.

We find that, as with the incompressible kinetic energy and decay of vortex line density, the qualitative behavior of $f(r)$ and $I$ is largely unchanged by the choice of cutoff $k_{c}$. Filtering less modes only has the effect of introducing disorder into the quasicondensate field, slightly reducing the quantitative values of $f(r)$ and $I$.

\section{CONCLUSIONS}

Using classical field simulations, we have modeled the evolution of a finite-temperature homogeneous Bose gas from a nonequilibrium initial condition, through the formation of a turbulent tangle of vortex lines to the relaxation to a vortex-free state. By monitoring the vortex line density, the energy spectrum, and the velocity correlation function, we have determined that the superfluid turbulence created by this thermal quench process lacks the coherent structures and the Kolmogorov cascade process which are typical of ordinary (classical turbulence), and which have also been observed in superfluid helium when driven by propellers or towed grids, or flowing at high velocity along channels. 
Instead, thermally quenched turbulence has properties similar to another regime of superfluid turbulence called ultraquantum turbulence, observed in superfluid helium under certain driving conditions, which is less organized and more similar to a random flow.

As the vortex tangle relaxes, the vortex line density decays as the power law $t^{-1}$, consistent with measurements of the decay of ultraquantum turbulence in superfluid helium. The behavior is observed across all three different condensate fractions $(0.22,0.48$, and 0.77$)$ considered. We expect this behavior to hold for all nonzero condensate fractions, providing the vortex dynamics are dominantly hydrodynamical, that is, providing the typical intervortex spacing exceeds the vortex core size (for example, the angular frequency $\omega$ of a vortex-vortex pair of separation $d$ and circulation $\kappa$ agrees with the classical value $\omega=\kappa /\left(\pi d^{2}\right)$ only if $d \gtrsim 2 \xi$, and deviates substantially [49] for $d<2 \xi$ ). Outside of this hydrodynamical regime, the overlap of vortex cores can be expected to cause a significant departure from the behavior we report, although this is yet to be explored in the case of turbulent vortices.
While our work is based the idealized paradigm of an infinite homogeneous system, such a system can be approximated experimentally through the recent advent of quasihomogeneous Bose-Einstein condensates formed in boxlike traps $[3,50]$. Nonetheless, condensates are most commonly confined in harmonic potentials, and it would be interesting in the future to explore how the inhomogeneity affects the nature of the turbulence induced by the thermal quench.

Data supporting this publication are openly available under an Open Data Commons Open Database License [51].

\section{ACKNOWLEDGMENTS}

G.W.S acknowledges support from the Engineering and Physical Sciences Research Council, and N.G.P. acknowledges funding from the Engineering and Physical Sciences Research Council (Grant No. EP/M005127/1). This work made use of the facilities of N8 HPC Centre of Excellence, provided and funded by the N8 consortium and EPSRC (Grant No. $\mathrm{EP} / \mathrm{K} 000225 / 1)$. The Centre is co-ordinated by the Universities of Leeds and Manchester.
[1] M. J. Davis, T. M. Wright, T. Gasenzer, S. A. Gardiner, and N. P. Proukakis, in Universal Themes of Bose-Einstein Condensation, edited by D. W. Snoke, N. P. Proukakis, and P. B. Littlewood (Cambridge University Press, Cambridge, UK, 2017).

[2] C. N. Weiler, T. W. Neely, D. R. Scherer, A. S. Bradley, M. J. Davis, and B. P. Anderson, Nature (London) 455, 948 (2008).

[3] L. Chomaz, L. Corman, T. Bienaime, R. Desbuquois, C. Weitenberg, S. Nascimbene, J. Beugnon, and J. Dalibard, Nat. Commun. 6, 6162 (2015)

[4] G. Lamporesi, S. Donadello, S. Serafini, F. Dalfovo, and G. Ferrari, Nat. Phys. 9, 656 (2013); S. Donadello, S. Serafini, M. Tylutki, L. P. Pitaevskii, F. Dalfovo, G. Lamporesi, and G. Ferrari, Phys. Rev. Lett. 113, 065302 (2014).

[5] T. W. B. Kibble, J. Phys. A 9, 1387 (1976).

[6] W. H. Zurek, Nature (London) 317, 505 (1985).

[7] B. V. Svistunov, J. Mosc. Phys. Soc. 1, 373 (1991).

[8] Yu. Kagan, B. V. Svistunov, and G. V. Shlyapnikov, Sov. Phys. JETP 74, 279 (1992).

[9] Yu. Kagan, B. V. Svistunov, and G. V. Shlyapnikov, Sov. Phys. JETP 75, 387 (1992).

[10] Yu. Kagan and B. V. Svistunov, Sov. Phys. JETP 78, 187 (1994).

[11] Yu. Kagan and B. V. Svistunov, Phys. Rev. Lett. 79, 3331 (1997).

[12] M. J. Davis, S. A. Morgan, and K. Burnett, Phys. Rev. Lett. 87, 160402 (2001).

[13] N. G. Berloff and B. V. Svistunov, Phys. Rev. A 66, 013603 (2002).

[14] M. J. Davis, S. A. Morgan, and K. Burnett, Phys. Rev. A 66, 053618 (2002).

[15] C. Connaughton, C. Josserand, A. Picozzi, Y. Pomeau, and S. Rica, Phys. Rev. Lett. 95, 263901 (2005).

[16] See Supplemental Material at http://link.aps.org/supplemental/ 10.1103/PhysRevA.94.053632 for a movie showing a sample evolution of the turbulent vortex tangle as it decays to equilibrium.

[17] J. Maurer and P. Tabeling, Europhys. Lett. 43, 29 (1998).
[18] J. Salort et al., Phys. Fluids 22, 125102 (2010).

[19] C. F. Barenghi, V. S. L'vov, and P.-E. Roche, Proc. Nat. Acad. Sci. USA 111, 4683 (2014).

[20] P. M. Walmsley and A. I. Golov, Phys. Rev. Lett. 100, 245301 (2008).

[21] D. E. Zmeev, P. M. Walmsley, A. I. Golov, P. V. E. McClintock, S. N. Fisher, and W. F. Vinen, Phys. Rev. Lett. 115, 155303 (2015).

[22] M. Brewczyk, M. Gajda, and K. Rzażewski, J. Phys. B 40, R1 (2007).

[23] N. P. Proukakis and B. Jackson, J. Phys. B 41, 203002 (2008).

[24] P. B. Blakie, A. S. Bradley, M. J. Davis, R. J. Ballagh, and C. W. Gardiner, Adv. Phys. 57, 363 (2008).

[25] S. Nazarenko, M. Onorato, and D. Proment, Phys. Rev. A 90, 013624 (2014).

[26] M. J. Davis and P. B. Blakie, Phys. Rev. Lett. 96, 060404 (2006).

[27] T. M. Wright, N. P. Proukakis, and M. J. Davis, Phys. Rev. A 84, 023608 (2011).

[28] A. Sinatra, C. Lobo, and Y. Castin, Phys. Rev. Lett. 87, 210404 (2001).

[29] M. Leadbeater et al., J. Phys. B 36, L143 (2003).

[30] T. P. Simula and P. B. Blakie, Phys. Rev. Lett. 96, 020404 (2006).

[31] G. W. Stagg, R. W. Pattinson, C. F. Barenghi, and N. G. Parker, Phys. Rev. A 93, 023640 (2016).

[32] N. G. Berloff and A. J. Youd, Phys. Rev. Lett. 99, 145301 (2007).

[33] B. Nowak, J. Schole, D. Sexty, and T. Gasenzer, Phys. Rev. A 85, 043627 (2012).

[34] N. G. Berloff and C. Yin, J. Low Temp. Phys. 145, 187 (2006).

[35] H. Salman and N. G. Berloff, Physica D (Amsterdam, Neth.) 238, 1482 (2009).

[36] R. W. Pattinson, N. P. Proukakis, and N. G. Parker, Phys. Rev. A 90, 033625 (2014).

[37] This can be understood by considering the largest possible vector in a three-dimensional box, where each dimension of the box is of length $k_{\text {nyq }}=f_{s} / 2=\pi / d$, the Nyquist frequency in 1D. The resulting vector is of size $k_{\max }=\sqrt{3} \pi / d$. 
[38] A. C. Newell, S. Nazarenko, and L. Biven, Physica D (Amsterdam, Neth.) 152, 520 (2001).

[39] C. Nore, M. Abid, and M. E. Brachet, Phys. Rev. Lett. 78, 3896 (1997).

[40] M. R. Smith, R. J. Donnelly, N. Goldenfeld, and W. F. Vinen, Phys. Rev. Lett. 71, 2583 (1993).

[41] S. R. Stalp, L. Skrbek, and R. J. Donnelly, Phys. Rev. Lett. 82, 4831 (1999).

[42] W. F. Vinen and J. J. Niemela, J. Low Temp. Phys. 128, 167 (2002).

[43] G. Volovik, JETP Lett. 78, 533 (2003).

[44] A. W. Baggaley, J. Laurie, and C. F. Barenghi, Phys. Rev. Lett. 109, 205304 (2012).
[45] A. W. Baggaley, C. F. Barenghi, and Y. A. Sergeev, Phys. Rev. B 85, 060501(R) (2012).

[46] M. Tsubota, T. Araki, and S. K. Nemirovskii, Phys. Rev. B 62, 11751 (2000).

[47] P. Walmsley, D. Zmeev, F. Pakpour, and A. I. Golov, Proc. Natl. Acad. Sci. USA 111, 4691 (2014).

[48] P. A. Davidson, Turbulence (Oxford University Press, Oxford, UK, 2004).

[49] N. G. Parker, Ph.D. thesis, University of Durham, United Kingdom, 2004, Fig. 8.2.

[50] A. L. Gaunt, T. F. Schmidutz, I. Gotlibovych, R. P. Smith, and Z. Hadzibabic, Phys. Rev. Lett. 110, 200406 (2013).

[51] Newcastle University Data, doi:10.17634/101785-3. 